\title{
Hiperplasia mamária felina: por que é tão comum no Brasil?
}

\author{
Feline mammary hyperplasia: why is it so common in Brazil? \\ Hiperplasia mamaria felina: ¿por que és tan común em Brasil?
}

Recebido: 13/04/2021 | Revisado: 25/04/2021 | Aceito: 27/04/2021 | Publicado: 10/05/2021

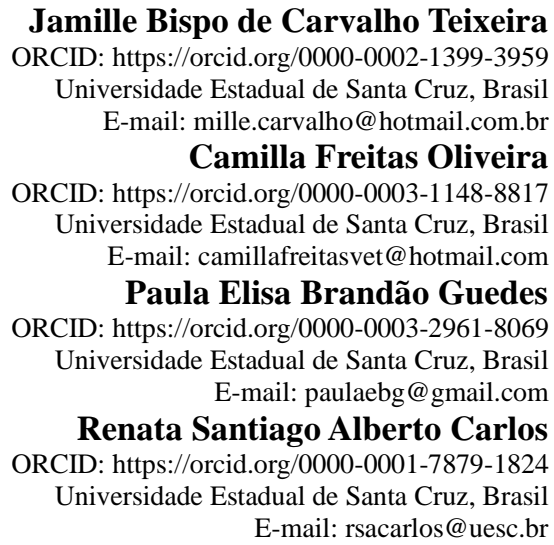

\begin{abstract}
Resumo
A Hiperplasia mamária felina (HMF) é uma alteração não neoplásica, caracteriza pela proliferação celular do estroma do ducto das glândulas mamárias, comumente diagnosticada no Brasil. Tal alteração muitas vezes é causada pelo uso inadequado de progestágenos exógenos em gatos, que podem ser armazenados no organismo do animal, e sensibilizam as glândulas à proliferação exacerbada por estímulo hormonal. Um dos fatores associados aos casos recorrentes da HMF é a venda desses medicamentos em lojas que prestam serviço de venda de produtos veterinários, aplicados sem restrições e sem conhecimento da fisiologia felina, na tentativa de controle gestacional. A conscientização de tutores sobre os malefícios da aplicação sem critério de progestágenos e a estimulação dos mesmos para que optem por esterilização cirúrgica dos seus animais deve ser feita pelos médicos veterinários, a fim de que se evitem maiores complicações como neoplasia mamária, garantindo, portanto, maior bem estar da população felina. $\mathrm{O}$ objetivo dessa revisão é abordar aspectos epidemiológicos, fisiológicos e clínicos, e promover alusão ao uso indiscriminado de anticoncepcionais nessa espécie.
\end{abstract}

Palavras-chave: Endocrinologia; Gatos; Tumor.

\begin{abstract}
Feline breast hyperplasia $(\mathrm{FBH})$ is a non-neoplastic disorder, characterized by cellular proliferation of the duct stroma of the mammary glands, commonly diagnosed in Brazil. Such change is often caused by the inappropriate use of exogenous progestogens in cats, which can be stored in the organism of the animal, and sensitize the glands to the proliferation exacerbated by hormonal stimulus. One of the factors associated with recurrent cases of FBH is the sale of these drugs in stores that provide service for the sale of veterinary products, applied without restrictions and without knowledge of feline physiology, in an attempt to control pregnancy. The awareness of owners about the harms of uncontrolled application of progestins and the stimulation of them to opt for surgical sterilization of their animals should be done by veterinarians, in order to avoid further complications such as breast cancer, thus ensuring greater welfare of the feline population. The purpose of this review is to address epidemiological, physiological and clinical aspects, and to promote an allusion to the indiscriminate use of contraceptives in this species.
\end{abstract}

Keywords: Cats; Endocrinology; Tumor.

\section{Resumen}

La Hiperplasia mamaria felina (HMF) es un trastorno no neoplásico, caracterizado por la proliferación celular del estroma del conducto de las glándulas mamarias, comúnmente diagnosticado en Brasil. Esta alteracione muchas veces es causada por el uso inadecuado de progestágenos exógenos en gatos, que pueden ser almacenados en el organismo del animal y sensibilizar las glândulas a la proliferación exacerbada por estímulos hormonales. Uno de los factores asociados a los casos recurrentes de HMF es la venta de estos medicamentos en tiendas que prestan servicio de venta de medicamentos veterinarios, aplicadas sin restricciones y sin conocimiento de la fisiología felina, en un intento por controlar la gestación. La sensibilización de tutores sobre los maleficios de la aplicación descontrolada de progestágenos y la estimulación de los mismos para que opten por esterilización quirúrgica de sus animales debe ser 
hecha por los médicos veterinarios, con el fin de evitar mayores complicaciones como neoplasia mamaria, garantizando por lo tanto mayor bienestar de la salud población felina. El propósito de esta revisión es abordar aspectos epidemiológicos, fisiológicos y clínicos y promover una alusión al uso indiscriminado de anticonceptivos en esta especie.

Palabras clave: Endocrinología; Tumor; Gatos.

\section{Introdução}

A hiperplasia mamária felina (HMF), também chamada de hiperplasia fibroepitelial felina (HFF), é caracterizada pela proliferação celular exacerbada do estroma do ducto de uma ou várias glândulas mamárias, e abrange $20 \%$ dos nódulos mamários em gatas (Hayden et al., 1989). O desenvolvimento da HMF é hormônio-dependente, principalmente da progesterona ou seus análogos, utilizados amplamente como anticoncepcionais em felinos (Gracanin et al., 2012; Loretti et al., 2005).

Além da hiperplasia, a administração do progestágeno exógeno na gata pode acarretar em outras afecções, como neoplasias mamárias, piometra, maceração fetal, ou até mesmo complicação no parto pelo relaxamento insuficiente da cérvix. Esses casos são bastante observados quando o anticoncepcional é aplicado em animais nas fases de estro, proestro e diestro (Keskin et al., 2009; Montanha et al., 2012; Souza et al., 2002).

Diante da importância para a sanidade dos felinos e da elevada casuística da HMF (Togni et al., 2013) se faz necessária a divulgação da problemática, no intuito de mobilização dos médicos veterinários, e o desenvolvimento de material de fácil acesso e compreensão, a fim de conscientizar e informar a população sobre os riscos do uso de anticoncepcionais nas gatas. O objetivo dessa revisão é abordar aspectos epidemiológicos, fisiológicos e clínicos, e promover alusão ao uso indiscriminado de anticoncepcionais na espécie felina.

\section{Metodologia}

Esse trabalho corresponde a uma revisão de literatura a respeito da hiperplasia mamária felina, realizada a partir de pesquisa bibliográfica sobre as principais informações necessárias para o entendimento dessa alteração, tais quais: fisiologia reprodutiva da gata, etiologia, sintomatologia, diagnóstico e tratamento, bem como uma discussão sobre o uso indiscriminado de contraceptivos e sua relação com a HMF. Para tanto, foram utilizadas bases de dados indexadas como Elsevier, Pubmed, Pubvet e Scielo.

\section{Revisão de literatura}

\subsection{Fisiologia reprodutiva da gata}

Como todo mamífero, a fêmea felina possui tecido glandular que secreta leite para alimentar sua prole. As glândulas mamárias, portanto, são compostas por tecido secretório circundado por tecido conectivo (Aspinall, 2011; Johnston et al., 2001).

A proliferação das glândulas mamárias em gatas ocorre na puberdade, marcada pelo primeiro ciclo estral, por volta dos cinco a sete meses de idade. Fêmeas felinas são naturalmente poliéstricas e possuem o ciclo estral denominado sazonal, ou seja, no hemisfério norte ciclam apenas em determinadas estações do ano, geralmente da primavera ao final do outono. Isso é uma exceção no caso das gatas de zonas com clima tropical, como no Brasil, que costumam ciclar durante todo o ano (Foss et al., 2013).

O ciclo estral da gata compreende basicamente cinco fases: proestro, estro, interestro, diestro e anestro (Feldman et al, 1996; Verhage et al., 1976). 
O proestro é a fase na qual se inicia o crescimento folicular e dura de um a dois dias (Feldman et al, 1996; Verhage et al., 1976).

Após o proestro, o animal entra na fase denominada estro, que compreende aproximadamente sete dias, com variação entre dois e 19 dias. Caracteriza-se pelo aumento da atividade folicular nos ovários, período no qual a fêmea apresenta sinais comportamentais característicos de cio e aceita a cópula (Feldman et al, 1996; Robinson et al., 2018). Vale ressaltar que a fêmea felina possui ovulação por indução, ou seja, necessita do estímulo da cópula para liberação dos óvulos e possível fecundação (Johnston et al., 2001; Wildt et al., 1981).

O interestro é uma fase caracterizada por aparecer após estros não-ovulatórios, em que há ausência de comportamento sexual e dura aproximadamente uma semana, podendo variar de um a 16 dias (Feldman et al, 1996; Johnston et al., 2001; Petersen, 2015).

O diestro ocorre após a ovulação. Neste caso, o corpo lúteo permanece em atividade secretando progesterona, hormônio que causa aumento da glândula mamária no estro e durante a gravidez, caso os óvulos sejam fecundados. Dura aproximadamente sessenta dias e termina com a luteólise. Caso não haja fecundação, o diestro pode resultar em uma pseudogestação (Feldman et al, 1996). Esse fenômeno é denominado pseudociese, que permanece por aproximadamente quarenta dias (Feldman et al, 1996; Johnston et al., 2001; Petersen, 2015).

Por fim, o anestro, cuja duração irá variar de acordo com a estação do ano, tem como característica principal o fim da atividade sexual da fêmea, no qual os níveis de progesterona e estrógeno são basais. Neste momento, há completa regressão do corpo lúteo e a gata não está receptiva ao macho (Feldman et al, 1996; Petersen, 2015). Esta fase pode não ocorrer em climas de constante fotoperíodo, como no Brasil, e a fêmea pode estar em período reprodutivo ininterrupto, apresentando gestações consecutivas (Petersen, 2015). Em outros casos, ocorre o denominado estro lactacional, no qual a fêmea apresenta cio em aproximadamente dez dias após o fim da lactação (Tsutsui et al., 1993).

\subsection{Etiologia e epidemiologia da hiperplasia}

Como citado anteriormente, a hiperplasia mamária felina é causada principalmente pela ação da progesterona ou seus análogos, como o acetato de medroxiprogesterona e acetato de megestrol (Romagnoli, 2015; Vigo et al., 2011). Tanto a progesterona produzida pelo próprio organismo do animal (endógena), quanto a oferecida por meio de injeções anticoncepcionais (exógena) podem levar ao aparecimento da afecção. No Brasil, a maioria dos casos está associada ao uso de anticoncepcionais, seja por via oral ou injetável (Filgueira et al., 2015).

A progesterona, exógena ou endógena, causa aumento da sensibilidade dos seus receptores, levando a uma resposta exacerbada de hormônio do crescimento das glândulas mamárias, culminando com processo hiperplásico (Gaviria et al., 2010; Krawczyk et al., 2017; Lopes et al., 2017). Dentre as gatas, acomete principalmente as fêmeas entre seis meses e treze anos de idade e não possui predisposição racial (Araújo et al., 2017; Gorlinger et al., 2002). As gatas não castradas são mais susceptíveis (Siberino et al., 2021). Fêmeas que estão na puberdade, durante o primeiro cio, gestantes ou que apresentam pseudociese, também estão entre as mais acometidas (Loretti et al., 2005). Em gatas castradas diagnosticadas com hiperplasia, a síndrome do ovário remanescente deve ser investigada (Gracanin et al., 2012).

Estudos mostram que gatas submetidas a apenas uma administração de um progestágeno sintético podem apresentar HMF (Loretti et al., 2005). Isso ocorre pelo acúmulo do hormônio exógeno, que pode ainda permanecer durante meses armazenado no organismo do animal, levando ao aparecimento de nódulos mamários (Robinson et al., 2018). De acordo esses trabalhos, os progestágenos podem aumentar em três vezes o risco do aparecimento da HMF, assim como lesões tumorais malignas (Aguiar et al., 2017). 
Há relatos de hiperplasia mamária também em machos que são tratados com progestágenos (Voorwald et al., 2021), para resolução de problemas comportamentais, dermatopatias, como granuloma eosinofílico e alopecia endócrina felina (MacDougall, 2003), ou incontinência urinária (Jacobs et al., 2010; MacDougall, 2003). Esse caso pode ser exemplificado por um relato de um gato de um ano de idade que apresentou hiperplasia mamária na mama inguinal. No artigo, o tutor do animal relatou que não houve aplicação equivocada ou intencional de alguma progestina no animal, mas essa possibilidade não pode ser descartada, já que o gato foi cuidado por pessoas distintas em tempos diferentes (Leidinger et al., 2011).

Outro caso de hiperplasia mamária em macho foi relatado, no qual o animal não recebeu nenhum tipo de progestágeno exógeno de seus tutores, nem foi tratado com qualquer medicamento que possuísse a substância. Suspeitou-se, portanto, de tecido ovariano em região testicular e/ou em região abdominal, mas os resultados das ultrassonografias e radiografia realizados descartaram essas possibilidades. Foi então solicitado exame sérico de progesterona, no qual as concentrações estavam em níveis normais. Há, portanto, a possibilidade de o animal ter entrado com contato com algum produto de uso humano contendo progestágeno em sua fórmula. Após o tratamento com aglepristone houve melhora rápida e eficaz, com completa remissão dos sinais clínicos (Mayayo et al., 2018).

Enquanto as hiperplasias mamárias são comumente observadas em animais que foram submetidos aos progestágenos por períodos mais curtos, o uso regular e prolongado dos anticoncepcionais, por mais de dez dias, é mais associado ao desenvolvimento de tumores mamários malignos, levando o animal a um prognóstico reservado (Giménez et al., 2010; Sorenmo et al., 2013).

\subsection{Endocrinologia do processo hiperplásico}

O controle de proliferação tecidual é delicadamente realizado por uma série de hormônios que emitem sinais de inibição ou replicação celular (Mol et al., 1995). O desenvolvimento da glândula mamária, portanto, não foge a essa regra e é influenciado por fatores de crescimento e hormônios que interagem entre si juntamente com os tecidos mesenquimais e epiteliais (Hovey et al., 2002), a saber:

a) Hormônio de Crescimento (GH)

O GH tem um papel importante na formação e desenvolvimento da glândula mamária. A glândula pituitária secreta formas lactogênicas e não lactogênicas de GH. Essas formas são mais potentes que a influência da prolactina sobre a proliferação do tecido mamário. O que acontece, portanto, é a produção desses reguladores de crescimento na glândula mamária da gata e cadela, sob influência das progestinas. Como resposta, o GH mamário recruta células tronco para diferenciarem-se em tecido glandular, mas essa diferenciação ocorre de forma precoce e as células se expandem no epitélio ductal sob influência de fatores de crescimento semelhantes à insulina (IGFs), dando origem ao processo hiperplásico (Mol et al., 1995; Rutteman, 1992).

\section{b) Estrogênio e Progesterona}

As células hiperplásicas felinas possuem receptores tanto para estrógeno quanto para progesterona, sugerindo que ambos os hormônios estão envolvidos nessa condição (Giménez et al., 2010). De acordo com estudos imuno-histoquímicos, os receptores de estrogênio (RE) podem estar presentes no tecido mamário de animais com hiperplasia, pois de acordo com um teste realizado, de dez animais que possuíam a afecção, em sete observou-se RE (Mulas et al., 2000a; Mulas et al., 2000b). Em outro estudo sobre a expressão de receptores de estrogênio e progesterona em tecido hiperplásico, observou-se que o receptor de estrogênio em células hiperplásicas chega a ser menor que a expressão em tecido normal, mas a expressão de progesterona foi significativamente maior nos casos de HFM (Millanta et al., 2006). 


\subsection{Achados clínicos}

À palpação, as lesões podem variar de um e meio a 18 centímetros de diâmetro, como massas firmes a macias e gelatinosas (Figuras 1, 2 e 3), podendo acometer uma, várias ou todas as mamas. Os animais comumente não apresentam dor, a menos que os nódulos estejam ulcerados ou inflamados (Amorim et al., 2006; Payan-Carreira, 2013; Rahal et al, 2003).

Sinais como edema, hiperemia e hipertermia são comumente encontradas nessas situações (Filgueira et al., 2008). O felino pode apresentar relutância ao caminhar, se as lesões forem demasiadamente extensas (Payan-Carreira, 2013). Devido a traumas e abcessos, podem ocorrer ulcerações por lambedura (Vasconcellos, 2003) ou por comprometimento de perfusão local. Isto pode culminar em processo isquêmico em situações crônicas, resultando em infecções bacterianas secundárias, que devem ser cuidadosamente tratadas com antibioticoterapia de amplo espectro (Araújo et al., 2017; Burstyn, 2010).

Figura 1: Hiperplasia em mama inguinal caudal direita (seta preta), consistência macia, medindo aproximadamente $4 \mathrm{~cm}$ de diâmetro.

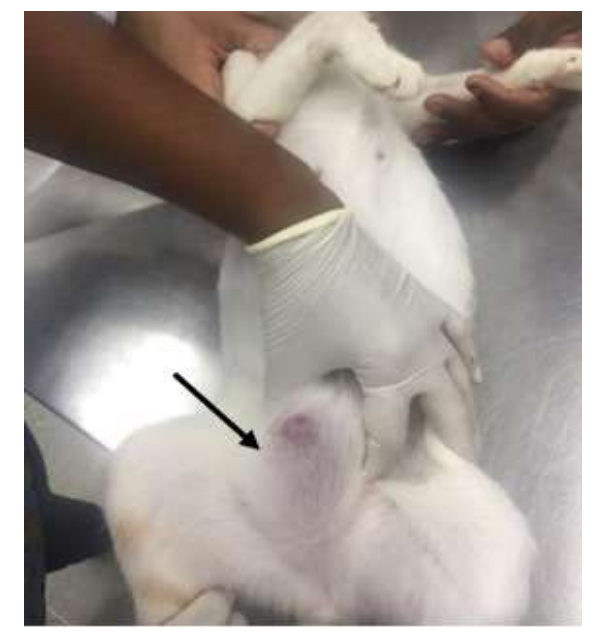

Fonte: Arquivo pessoal.

Figura 2: Hiperplasia bilateral em mamas torácicas e abdominais (setas pretas) de consistência firme apresentando aproximadamente $4 \mathrm{~cm}$ de diâmetro.

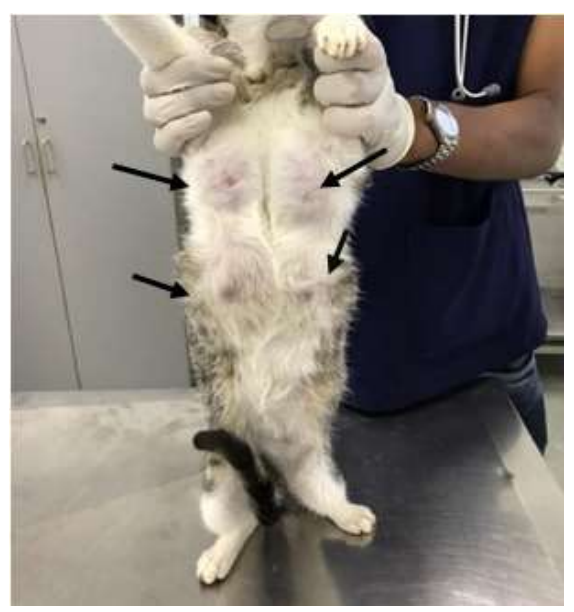

Fonte: Arquivo pessoal. 
Figura 3: (A) Hiperplasia ulcerada em mamas torácicas (setas azuis) e abdominais (setas vermelhas) de consistência firme, com aproximadamente $5 \mathrm{~cm}$ de diâmetro. (B) Hiperplasia ulcerada em mamas abdominais (seta preta) com consistência fïrme, com aproximadamente $7 \mathrm{~cm}$ de diâmetro.

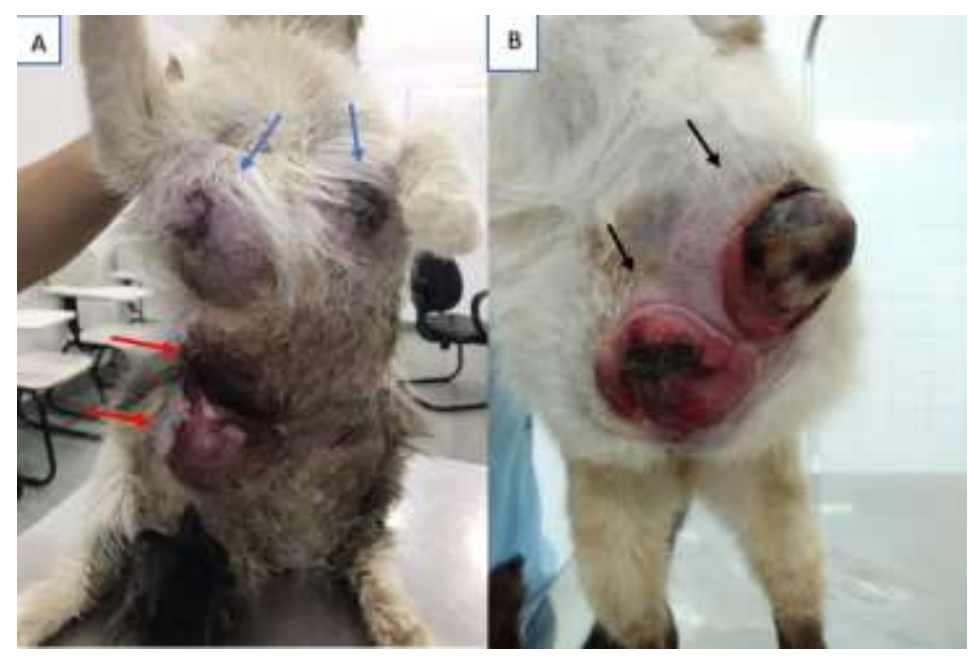

Fonte: Arquivo pessoal.

\subsection{Diagnóstico}

O diagnóstico da hiperplasia fibroadenomatosa felina é geralmente baseado no histórico do animal e investigação de administração irregular de progestágenos. $\mathrm{Na}$ inspeção das glândulas, pode ser observada ectasia dos vasos, assim como hipertermia ao toque e adelgaçamento da pele das mamas (Gaviria et al., 2010; Giménez et al., 2010; Payan-Carreira, 2013). Deve-se atentar também ao aparecimento das lesões em animais castrados submetidos a tratamentos de dermatopatias com progestágenos (Payan-Carreira, 2013), ou que sofrem de síndrome do ovário remanescente. Neste caso, a confirmação de cio deve ser diagnosticada através de citologia vaginal e observação comportamental (Giménez et al., 2010).

Em animais que apresentam produção de leite, a ultrassonografia (USG) abdominal pode ser realizada para descartar qualquer suspeita de gestação. Pode ser também realizada a USG dos tecidos mamários, que será de grande utilidade para a observação do tecido fibroepitelial, em que se nota, então, maior ecogenicidade quando comparada ao tecido normal. A radiologia não é usualmente utilizada para o diagnóstico de HMF, mas radiografias torácicas são recomendadas em casos de diagnósticos diferenciais de neoplasias, para que se descarte possíveis metástases (Payan-Carreira, 2013).

Dosagens de progesterona sérica podem ser realizadas para a constatação de altos níveis do hormônio no animal, que são compatíveis com hiperplasia mamária (Giménez et al., 2010). Contudo, quando a causa da HMF é por medicamento exógeno, a dosagem de progesterona pode estar em baixos níveis, devido à baixa sensibilidade deste método de diagnóstico (Payan-Carreira, 2013).

Ao exame citológico será observada proliferação fibroglandular, cujas células apresentam morfologia compatível com células normais da glândula mamária (Hayden et al., 1983) (figura 4A), embora possam estar presentes anisocariose e anisocitose moderadas, além de eosinofilia da matriz extracelular (Payan-Carreira, 2013).

Para realização de diagnóstico definitivo, a biópsia pode ser empregada (Amorim et al., 2006). A realização deste exame deve ser complementar ao histórico e sinais clínicos do animal. Biópsias excisionais e incisionais cirúrgicas podem ser realizadas e analisadas através da histopatologia (Amorim et al., 2006; Little, 2011; Loretti et al., 2005). A HFM possuirá um tecido semelhante à glândula mamária normal, com as principais diferenças nos tamanhos dos ductos, que estarão mais desenvolvidos e o estroma mais vascularizado que o tecido normal (Hayden et al., 1983) (figura 4B). 
Figura 4: (A) Citopatologia de hiperplasia mamária felina. Notar proliferação fibroglandular, cujas células apresentam morfologia compatível com células normais da glândula mamária (seta preta). 40 X. Diff-Quick. (B) Fotomicrografia de hiperplasia mamária. Notar proliferação de células epiteliais bem diferenciadas, morfologicamente similares às células normais dos ductos mamários e delimitadas por uma camada de células mioepiteliais (setas pretas). $40 \mathrm{X}$. HE.

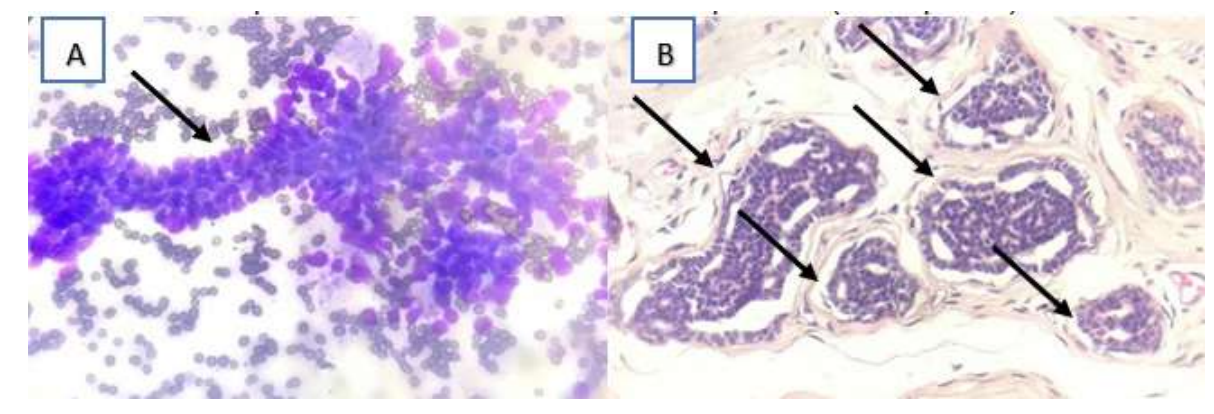

Fonte: Arquivo pessoal.

\subsection{Tratamento}

A hiperplasia é uma alteração benigna, no entanto, dependendo do estado clinico do paciente pode ser considerada emergencial. Pode ser instituído tratamento cirúrgico e/ou clinico (Loretti et al., 2005; Vasconcellos, 2003).

\section{a) Tratamento Cirúrgico}

A realização da ovariosalpingohisterectomia $(\mathrm{OSH})$ deve ser indicada após a involução total das mamas, no caso de hiperplasias de causa exógena, com o objetivo de evitar recidivas. Uma vez que com a OSH a fonte de progesterona endógena é retirada e o animal não mais apresentará ciclo estral, o uso de progesterona exógena por parte dos tutores também será evitado (Munson, 2006).

A OSH também é indicada quando a causa da hiperplasia é endógena, no intuito de se retirar a fonte primária hormonal. A OSH realizada antes da involução total das glândulas mamárias exige que o acesso cirúrgico seja pelo flanco, uma vez que pela linha média se torna inviável na maioria dos casos. Em situações em que a involução mamária não ocorra mesmo após a retirada do estímulo hormonal ou se observa recidiva (Viana et al., 2012), a mastectomia é indicada (Amorim et al., 2006).

A OSH apresenta resultados satisfatórios (Kutzler \& Wood, 2006; Silva \& Silva, 2012) principalmente quando a causa da hiperplasia mamaria é decorrente da produção de progesterona endógena, no entanto, os tutores devem estar conscientes que a OSH impossibilita a reprodução dessa fêmea. Devem ser levados em consideração os riscos inerentes a todo procedimento cirúrgico, e que gatos submetidos à castração também podem apresentar quadros de hiperplasia em decorrência da diminuição da concentração de testosterona. Essa redução pode promover alterações hormonais que culminam no surgimento da hiperplasia fibroadenomatosa felina (Estévez et al., 1999).

Embora a mastectomia não seja o tratamento mais adequado, tendo em vista que se tem disponíveis no mercado medicamentos efetivos, possibilitando protocolos terapêuticos menos invasivos (Amorim et al., 2006), deve ser cogitada quando se observa um déficit no aporte sanguíneo para o tecido mamário, culminando no surgimento de processos ulcerativos e necróticos (Nelson \& Couto, 2015). A excisão cirúrgica também permite a realização do exame histopatológico e diferenciação de outras alterações mamárias (Gomide, 2009).

\section{b) Tratamento Clínico}

Quando essa alteração é decorrente de fonte de progesterona exógena, o primeiro passo é suspender o uso do método contraceptivo utilizado (Romagnoli, 2015). Quando essa alteração é decorrente de fonte de progesterona endógena, usualmente 
opta-se pela OSH, conforme relatado anteriormente (Amorim et al., 2006). Em casos de ulcerações ou necrose tecidual, decorrentes do crescimento rápido da mama com comprometimento do aporte sanguíneo, se faz necessário o tratamento de suporte com o uso de anti-inflamatórios não-esteroidais, antibióticos de amplo espectro, analgésicos, bem como o uso da metergolina, antiprolactina (Araújo et al., 2017; Burstyn, 2010).

Mais recentemente tem se observado sucesso nas terapias com o uso do aglepristone (Nelson \& Couto, 2015; Uçmak et al., 2011), o qual vem sendo amplamente utilizado como tratamento em casos de hiperplasia mamária em gatas (Silva et al., 2012). Trata-se de um antiprogestágeno que se liga aos receptores da progesterona, bloqueando sua ação. Ele é indicado como abortivo e no tratamento de piometra em cadelas e gatas, apresentando alta eficácia. A dose é de $10 \mathrm{mg} / \mathrm{kg}$ por via subcutânea (Gogny \& Fieni, 2016; Vitasek et al., 2006; Wherend et al., 2001).

$\mathrm{Na}$ literatura foi descrito o uso do antiprogestágeno a cada 24 horas durante cinco dias consecutivos, não sendo relatados efeitos colaterais. A involução da glândula mamária foi observada um mês após a primeira aplicação (Jurka \& Max, 2009; Wherend et al., 2001). Além disso, há opção pela aplicação alternada entre os dias 1, 2, 7 e 21, com regressão total após seis semanas (Vitasek et al., 2006).

O uso do aglepristone na dose $20 \mathrm{mg} / \mathrm{kg}$, uma vez por semana ou na dose de $15 \mathrm{mg} / \mathrm{kg}$ duas vezes por semana em dias consecutivos, durante 1 a 4 semanas, por via subcutânea, já foi estabelecida. No entanto com esse protocolo foram observados efeitos colaterais como a endometrite e reação dermatológica no local da aplicação (Gorlinger et al., 2002).

Existem relatos em gatas que fizeram o uso de progestágenos cumulativos; nesses casos deve ser instituído um novo ciclo com a aglepristone, uma vez que esse fármaco se liga aos receptores do progestágeno, bloqueando sua ação, mas não os inativando, permitindo que novos picos do progestágeno na corrente sanguínea desencadeiem novos processos de hiperplasia mamária (Vasconcellos, 2003).

Um fator limitante para o uso do antiprogestágeno ainda é o seu custo elevado, encarecendo o tratamento e dificultando o seu uso. Um frasco de $10 \mathrm{ml}$ do produto pode custar em média $\mathrm{R} \$ 394,90$. (Alizin $10 \mathrm{ml}$, Virbac, na Magazine Luiza em maio de 2020; disponível em: <https://www.magazineluiza.com.br/alizin-10->).

\subsection{Porque ainda temos tanto problema com o uso de anticoncepcional no Brasil?}

O uso de anticoncepcionais injetáveis em cães e gatos se iniciou na década de 80 e o seu uso ainda é frequente nos dias atuais. Apesar da eficácia do método cirúrgico para esterilização das fêmeas, muitos tutores visando à contenção de gastos ou por falta de informação, optam pela aplicação dos progestágenos como método contraceptivo. Essa é uma observação que fazemos com alta frequência na rotina de atendimentos clínicos na região na qual estamos inseridos. Dentre os 355 (185 fêmeas e 170 machos) pacientes felinos atendidos com queixas clínicas no Departamento de Clínica de Pequenos Animais do Hospital Veterinário da Universidade Estadual de Santa Cruz em Ilhéus, Bahia, nos anos de 2017, 2018 e 2019, 23 das 185 gatas $(12,4 \%)$ foram diagnosticadas com hiperplasia mamária, e todos esses animais foram submetidos ao uso de progestágenos exógenos, segundo relato dos tutores.

A administração oral ou injetável de progestágenos sintéticos como o acetato de medroxiprogesterona e acetato de megestrol (Romagnoli, 2015; Vigo et al., 2011) é facilitada, uma vez que são amplamente comercializados em lojas que prestam serviço de venda de medicamentos veterinários. Essas drogas são vendidas sem receita médica e aplicadas por pessoas não capacitadas ou pelo tutor do animal, e podem aumentar o número de casos de hiperplasia mamária felina muitas vezes por dosagem inapropriada.

As gatas, conforme citado anteriormente, atingem a maturidade sexual aos seis meses de idade e no Brasil apresentam o ciclo estral durante o ano inteiro. Além disso, o intervalo entre os ciclos estrais pode ser curto (duas semanas), gerando proles sequenciais, muitas vezes numerosas (Thompson, 2004). Essas características dificultam o controle populacional (Honório et 
al., 2017) fazendo com que os tutores optem pelo uso dos anticoncepcionais para controlar o ciclo estral, justamente pelo fácil acesso aos mesmos e o baixo custo quando comparado com o procedimento cirúrgico eletivo, a OSH (Aguiar et al, 2017; Oliveira \& Marques, 2006).

Deve-se levar em consideração que muitos tutores desconhecem os malefícios do uso dos progestágenos e em muitas vezes não possuem fácil acesso ao acompanhamento médico veterinário para seus animais. Estes fatores levam ao uso de medicamento sem prescrição médica, culminando no comprometimento da saúde e bem-estar dos animais (Aguiar et al., 2017; Pantoja et al. 2017; Silva et al., 2020).

\section{Considerações Finais}

A hiperplasia mamária em gatas é bastante recorrente na rotina de atendimento clínico, devido, principalmente, ao uso indiscriminado de progestágenos exógenos. Apesar dessa condição clinica apresentar caráter benigno, podem-se observar manifestações graves que podem levar ao óbito do paciente.

Dessa forma, os contraceptivos hormonais utilizados para bloquear temporariamente a fertilidade dos felinos apresentam riscos, por produzirem efeitos colaterais graves para a saúde reprodutiva e geral dos animais, como a hiperplasia mamária felina. Assim, mais estudos que testem e comprovem a segurança e eficácia dos contraceptivos em gatas são necessários, a fim de se conhecer os benefícios e malefícios do uso de anticoncepcionais em felinos domésticos.

Atualmente, o aglepristone vem apresentando resultados significativos no tratamento da HMF, demonstrando ser um tratamento seguro, eficaz e bem tolerado pelos animais, permitindo a redução do número de animais submetidos à mastectomia, porém mostra-se um tratamento de alto custo.

A castração é a melhor opção na prevenção da hiperplasia mamária em gatos. Em alguns locais são realizadas campanhas e mutirões a fim de esterilizar a maior quantidade de animais possível, evitando essa e outras afecções, além de realizar o controle de natalidade dos animais (Munson, 2006).

\section{Referências}

Aguiar, R. A. C., Moreira, V. S. \& Porto, M. R. (2017). Patologias Reprodutivas diagnosticadas durante a ovariosalpingoesterectomia (OSH) em gatas e cadelas. Simpósio de TCC e seminário de IC. 2016. Disponível em 28 Congresso Nacional de Medicina Veterinária FAG.

Amorim, F. V., Souza, H. J. M., Ferreira, A. M. R. \& Fonseca, A. B. M. (2006). Clinical, cytological and histopathological evaluation of mammary masses in cats from Rio de Janeiro, Brazil. Journal of Feline Medicine and Surgery, 8, 379-388.

Araújo, E. K. D., Moura, V. M., Honório, T. G. A. F., Alves, R. A., Fonseca, A. P. B., Rodrigues, M. C. \& Klein, R. P. (2017). Principais patologias relacionadas aos efeitos adversos do uso de fármacos contraceptivos em gatas em Teresina - PI. Pubvet, 11 (3), $256-261$.

Aspinall, V. (2011). Reproductive system of the dog and cat part 1 - the female system. Veterinary Nursing Journal, 26, 43-55.

Burstyn, U. (2010). Management of mastitis and abscessation of mammary glands secondary to fibroadenomatous hyperplasia in a primiparturient cat. Journal of the American Veterinary Medical Association, 236 (3), 326-329.

Estévez, J. O., De La Sota, R. L. \& Gobello, M. C. (1999). Fibroadenomatous mammary hyperplasia in a neutered cat. Revista Brasileira de Reprodução animal, 26 (3), 470-496.

Feldman, E. C. \& Nelson, R. W. (1996). Breeding Pregnancy and Parturition. In: Feldman, E. C. \& Nelson, R. W. Canine and Feline endocrinology and Reproduction. Philadelphia: Saunders, 547-571.

Filgueira, K. D., Reis, P. C. C. R. \& Paula, V. V. (2008). Relato de caso: hiperplasia mamária felina: sucesso terapêutico com o uso do aglepristone. Ciência Animal Brasileira, 9 (4), 1010-1016.

Filgueira, K. D., Reis, P. C. C. R., Macêdo, L. B., Oliveira, I. V. P. M., Pimentel, M. M. L., \& Júnior. A. R. (2015). Caracterização clínica e terapêutica de lesões mamárias não neoplásicas em fêmeas da espécie felina. Acta Veterinaria Brasilica, 9 (1), 98-107.

Foss, M. A., County, S., Stwart, N., County, K. \& County, J. S. S. (2013). Cat Anatomy and Physiology. Washington State University.

Gaviria, E. F. B., Bonilla, D. F. E. \& Gómez, A. F. L. (2010). Hiperplasia fibroepitelial mamaria felina: reporte de un caso. Revista CES Medicina Veterinaria y Zootecnia, 5 (1), 70-76. 
Giménez, F., Hecht, S., Craig, L. E. \& Legendre, A. M. (2010). Early detection, agressive therapy: Optimizing the management of feline mammary masses. Journal of Feline Medicine and Surgery, 12, 214-224.

Gogny, A. \& Fieni, F. (2016). Aglepristone: A review on its clinical use in animals. Theriogenology, 85 (4), 555-566.

Gomide P. R. (2009). Estudo retrospectivo de cadelas portadoras de neoplasia mamáriasubmetidas à mastectomia. Faculdade de Medicina Veterinária e Zootecnia (FMVZ), Universidade Estadual Paulista (Unesp), Botucatu.

Gorlinger, S., Kooistra H. S., Van Den Broek, A. \& Okkens, A. C. (2002). Treatment of fibroadenomatous hyperplasia in cats with aglépristone. Journal of Veterinary Internal Medicine, 16, 710-713.

Gracanin, A., De Gier, J., Zegers, K., Bominaar, M., Rutteman, G. R., Schaefers-Okkens, A. C., Kooistra, H. S. \& Mol. J. A. (2012). Progesterone Receptor Isoforms in the Mammary Gland of Cats and Dogs. Reproduction in Domestic Animals, 47 (6), 313-317.

Hayden, D. W., Johnson, K. H. \& Ghobrial, H. K. (1983). Ultrastructure of Feline Mammary Hypertrophy. Veterinary Pathology, $20,254-264$.

Hayden, D. W., Barnes, D. M. \& Johnson, K. H. (1989). Morphologic changes in the mammary gland of megestrol acetate-treated and untreated cats: A retrospective study. Veterinary Pathology, 26, 104-103.

Honório, T. G. A. F., Fonseca, A. P. B., Araújo, E. K. D., Moura, V. M., Chaves, R. A. A., Rodrigues, M. C. \& KLEIN, R. P. (2017). Implicações patológicas após o uso de anticoncepcional, em cadelas situadas em Teresina - PI. Pubvet, 11 (2), 103-206.

Hovey, R. C., Trott, J. F. \& Vonderhaar, B. K. (2002). Establishing a framework for the functional mammary gland: From endocrinology to morphology. Journal of Mammary Gland Biology and Neoplasia, 7 (1), 17-38.

Jacobs, T. M., Hoppe, B. R., Poehlmann, C. E., Ferracone, J. D. \& Sorenmo, K. U. (2010). Case report: Mammary adenocarcinomas in three male cats exposed to medroxyprogesterone acetate (1990-2006). Journal of Feline Medicine and Surgery, 12 (2), 169-174.

Johnston, S. D., Kustritz, M. V. R. \& Olson, P.N.S. (2001). Canine and Feline Theriogenology. Saunders.

Jurka, P. \& Max, A. (2009). Treatment of fibroadenomatosis in 14 cats with aglepristone - changes in blood parameters and follow-up. The Veterinary Record, $165,657-660$

Keskin, A., Yilmazbas, G., Yilmaz, R., Ozyigit, M. O. \& Gumen, A. (2009). Pathological abnormalities after long-term administration of medroxyprogesterone acetate in a queen. Journal of Feline Medicine and Surgery, 11 (6), 518-521.

Krawczyk M., Łopuszyński, W., Szczubiał, M. \& Millán, Y. (2017). Progesterone receptor expression and proliferative activity in relation to histological architecture of feline mammary fibroadenomatous change. Medycyna Weterynaryjna-Veterinary Medicine-Science and Practice, 73 (1), 33 - 38.

Kutzler, M. \& Wood, A. (2006). Non-surgical methods of contraception and sterilization. Theriogenology, 66 (3), 514-525.

Leidinger, E., Hooijberg E., Sick, K., Reinelt, B. \& Kirtz, G. (2011). Fibroepithelial hyperplasia in an entire male cat: Cytologic and histopathological features. Tierärztliche Praxis, 39 (3), 198-202.

Little, S. (2011). Feline Reproduction: Problems and clinical challenges. Journal of Feline Medicine and Surgery, 13, 508-515.

Lopes, M. D. \& Ackermann, C. L. (2017). Contracepção em felinos domésticos: novas abordagens. Revista Brasileira de Reprodução Animal, 41 (1), $270-277$.

Loretti, A.P., Ilha, M. R. S., Ordás, J. \& Mulas, J M. (2005). Clinical, pathological and immunohistochemical study of feline mammary fibroepithelial hyperplasia following a single injection of depot medroxyprogesterone acetate. Journal of Feline Medicine and Surgery, 7 (1), 43-52.

MacDougall, L. D. (2003). Mammary fibroadenomatous hyperplasia in a young cat attributed to treatment with megestrol acetate. Canadian Veterinary Journal, 44, 227-229.

Mayayo, S. R., Bo, S. \& Pisu, M. C. (2018). Mammary fibroadenomatous hyperplasia in a male cat. Journal of Feline Medicine and Surgery Open Reports, 4 (1), $1-5$.

Millanta, F., Calandrella, M., Vannozzi, I. \& Polli, A. (2006). Steroid hormone receptors in normal, dysplastic and neoplastic feline mammary tissues and their prognostic significance. The Veterinary Record, 158, 821-824.

Mol, J. A., Garderen, E., Selman, P. J., Wolfswinkel, J., Rijnberk, A. \& Rutteman, G. R. (1995). Growth Hormone mRNA in Mammary Gland Tumors of Dogs and Cats. Journal of Clinical Investigation, 95, 2028-2034.

Montanha, F. P., Corrêa, C. S. S. \& Parra, T. C. (2012). Maceração fetal em gata em decorrência do uso de contraceptivos - relato de caso. Revista Científica Eletrônica de Medicina Veterinária, 10 (19), 1-6.

Mulas, J. M., Niel, M., Millán, Y., Blankenstein, M. A., Mil, F. \& Misdorp, W. (2000a). Immunohistochemical analysis of estrogen receptors in feline mammary gland benign and malignant lesions: comparison with biochemical assay. Domestic Animal Endocrinology, 18 (1), 111-125.

Mulas, J. M., Millán, Y., Bautista, J. M., Pérez, J. \& Carrasco, L. (2000b). Oestrogen and progesterone receptors in feline fibroadenomatous change: an immunohistochemical study. Research in Veterinary Science, 68 (1), 15-21.

Munson, L. (2006). Contraception in felids. Theriogenology, 66 (1), 126-134.

Oliveira, E. C. S. \& Marques J. R. A. P. (2006). Endocrinologia reprodutiva e controle da fertilidade da cadela. Revista Brasileira de Reprodução Animal, 30 $(1 / 2), 11-18$. 
Nelson, R. W. \& Couto, C. G. (2015). Medicina Interna de Pequenos Animais. Elsevier: Amsterdan.

Pantoja, A. R., Bastos, M. M. S. \& João, C. F. (2017). Hiperplasia mamária felina. Ciência Animal, 27 (3), 89-98.

Payan-Carreira, R. (2013). Feline Mammary Fibroepithelial Hyperplasia: A Clinical Approach. In: Insights from veterinary medicine. InTechOpen, 215-232.

Petersen, A. (2015). Reproductive Physiology of the Female Cat. Dissertação (Mestrado em Medicina Veterinária). Fakulteten för veterinärmedicinoch husdjursvetenskap, Swedish University of Agricultural Sciences, Upsala.

Rahal, S. C., Caporali, E. H. G., Lopes, M. D., Rocha, N. S. \& Melero, F. H. (2003). Hiperplasia mamária felina - relato de três casos. Ars Veterinária, 19 (2), $188-190$.

Romagnoli, S. (2015). Progestins to control feline reproduction: Historical abuse of high doses and potentially safe use of low doses. Journal of Feline Medicine and Surgery, 17 (9), 743-752.

Robinson, B. \& Noakes, D. E. (2018). Reproductive physiology of the female. In: Noakes, D. E., Pearson, H., Parkinson, T. J. \& England, G. Veterinary Reproduction \& Obstetrics. Beijing: Elsevier, 2 - 35.

Rutteman, G. R. (1992). Contraceptive steroids and the mammary gland: Is there a hazard? - Insights from animal studies. Breast Cancer Research and Treatment, 23, 29-41.

Siberino, G. B., Ortiz, B. C., Matte, K. L., Secchi, P., Gomes, V. R., Pozzatti, D. \& Figueiredo, K. G. (2021). Hiperplasia mamária felina: Relato de caso. PubVet, 15 (1), 1-4.

Silva, F. B., Lima, A. E. S., Ribeiro, L. G. R., Neto, J. M. C., Costa, A. C. F., Silveira, C. P. B., Mello, S. B., Larangeira, D. F. \& Oriá, A. P. (2012). Utilização do aglepristone no tratamento da hiperplasia fibroepitelial mamária felina. Veterinária e Zootecnia, 19 (3), 399-406.

Silva, T. P. D. \& Silva, F. L. (2012). Hiperplasia mamária felina: Um relato de caso. Enciclopédia Biosfera, Centro Científico Conhecer, 8 (14), 634-640.

Silva, F. L., Silva, C. R. A., Sousa, M. P., Castro, L. R. M. S., Rocha, A. O., Costa, T. M., Brito, T. K. P., Fernandes, E. R. L. \& Rodrigues, K. E. R. (2020). Avaliação do uso de anticoncepcionais em cães e gatos. PubVet, 14 (10), 1-5.

Sorenmo, K. U., Worley, D. R. \& Goldschmidt, M. H. (2013). Tumors of the Mammary Gland. In: Withrow, S. J., Vail, D. M., Page, R. L. (Ed.) Small Animal Clinical Oncology. Missouri: Saunders, 540-556.

Souza, T. M., Fighera, R. A., Langohr, I. M. \&Barros, C. S. L. (2002). Hiperplasia fibroepitelial mamária em felinos: cinco casos. Ciência Rural, 32 (5), 891 894.

Thompson, F. N. Reprodução em mamíferos do sexo feminino. In: Reece, W. O. \& DUKES. (2004). Fisiologia dos animais domésticos, (12a ed.), Guanabara Koogan, 644-669.

Togni, M., Masuda, E. K., Kommers, Fighera, R. A. \& Irigoyen, L. F. (2013). Estudo retrospectivo de 207 casos de tumores mamários em gatas. Pesquisa Brasileira Veterinária, 33 (3), 353-358.

Tsutsui, T. \& Stabenfeldt, G. H. (1993). Biology of ovarian cycles, pregnancy and pseudopregnancy in the domestic cat. Journal of Reproduction and Fertility, 47, 29- 35

Uçmak, M., Enginler, S.Ö., Gündüz, M. C., Kirşan, I. \& Sönmez, K. (2011). Treatment of feline mammary fibroepithelial hyperplasia with the combination of aglepristone and cabergoline. Journal of The Faculty of Veterinary Medicine, 37 (1), 69-73.

Vasconcellos, C. H. C. Hiperplasia mamária In: Souza, H. J. M. (2003). Coletâneas em medicina e cirurgia felina. L. F. Livros, 23 - 237.

Verhage, H. G., Beamer, N. B. \& Brenner, R. M. P. (1976). Plasma levels of estradiol and progesterone in the cat during polyestrus, pregnancy and pseudopregnancy. Biology of Reproduction, 14, 579-585.

Viana, D. C., Santos, A. C., Rui, L. A., Oliveira, D. M., Silva, A. B., Costa, F. C. F. C. \& Neto, A. C. A. (2012). Hiperplasia mamária felina-relato de caso. Veterinária Notícias, 18 (2), 121-125.

Vigo, F., Lubianca, J. N. \& Corleta, H. E. (2011). Progestógenos: farmacologia e uso clínico. Femina, 39 (3), $127-137$.

Vitasek, R. \& Dendisova, H. (2006). Treatment of feline mammary fibroepithelial hyperplasia following a single injection of proligestone. Acta Veterinaria, 75 (2), 295-297.

Voorwald, F. A., Lopes, C., Silveira, G. C., Lima, D. T., Silva, M. F. C., Andreão, N. B. \& Toniollo, G. H. (2021). Severe mammary fibroepithelial hyperplasia due to single injection of medroxyprogesterone acetate in two male cats. Ciência Rural, 51 (4), 1-9.

Wherend, A., Hospes, R. \& Gruber, A. D. (2001). Treatment of feline mammary fibroadenomatous hyperplasia with a progesterone antagonist. Veterinary Records, 148 (11), 346-347.

Wildt, D. E., Chan, S. Y. W., Seager, S. W. J. \& Chakraborty, P. K. (1981). Ovarian activity, circulating hormones and sexual behaviot in the cat. Relationships during the coitus induced luteal phase and the estrous period without mating. Biology of Reproduction, $25,15-18$. 